\title{
False Claims: A Case Report of a Questionable Head Injury
}

\author{
Luv Sharma ${ }^{1 *}$, Ishwar Singh ${ }^{2}$ and Jyotsna Sen ${ }^{3}$ \\ ${ }^{1}$ Department of Forensic Medicine and MD, Professor, University of Health Sciences, Haryana, India \\ ${ }^{2}$ Department of Neuro-Surgery Senior Professor and Head University of Health Sciences, Haryana, India \\ ${ }^{3}$ Department of Ratio-diagnosis Senior Professor University of Health Sciences, Haryana, India
}

*Corresponding author: Dr. Luv Sharma, Department of Forensic Medicine and MD, Professor, 24/9-J, Medical Enclave, Pt. BD Sharma Post-Graduate Institute of Medical Sciences, University of Health Sciences, Rohtak124001, Haryana, India. Tel: 911262213165,919416101258, E-mail: drluvksharma@yahoo.com

Citation: Luv Sharma, Ishwar Singh, Jyotsna Sen (2017) False Claims: A Case Report of a Questionable Head Injury J Forensic Cri Sci 1: 104

Article history: Received: 17 January 2017, Accepted: 30 May 2017, Published: 02 June 2017

\begin{abstract}
Fabricated injury is a major problem for forensic experts. Such injuries are also termed as feigned/fictitious/ simulated injuries involving pretense of the patient to present with an injury. We present an interesting case of such a simulated injury in which the patient presented an old surgical craniotomy flap as an entirely new injury. The case was referred to our Institute from a peripheral hospital where the medico-legal report described two incised wounds on the scalp; the primary radiological investigations were reported hemorrhagic contusion and fracture skull. A second examination including a new CT-scan was ordered by the courts at our center. The presence of a healed curvilinear surgical scar and craniotomy flap with burr holes in both the CT-scans were conclusive in detecting feigned injuries by the patient. This case adds to the existing knowledge of fabricated injuries where the patient presented an old surgical scar and craniotomy as an entirely new injury.
\end{abstract}

Keywords: Simulated; Injuries; Radiology; Head Surgery

\section{Introduction}

Fabricated, fictitious, forged or invented wounds are those which may be produced by a person on his own body (self-inflicted) or occasionally, caused by another person with his consent (self-suffered) [1]. The most common is fabrication of an injury to simulate an assault. The underlying motives for fabricated injuries vary from arousal of sympathy to that of hate. There are several etiological theories for self-harm behaviour such as past trauma, invalidation, sexual and physical abuse, neglect and neuro-chemical changes $[2]$.

Luber et al report that certain clinical conditions may be mis-diagnosed as sequelae of trauma. They state that the classic presentation of hypoglycemia, a patient with diabetes mellitus on medical therapy with an altered sensorium can lead the physician to assume that the alteration in consciousness has resulted from a head injury and not a metabolic disorder [3].

Gentleman has commented that as head injury is the result of mechanical forces being applied to the head, the most widely used early indices of severity are conscious level disturbance, post-traumatic amnesia, and structural damage (as seen on a CT or MRI brain scan). Each of these methods can help to predict early and late outcome after head injury, and the information they provide is additive [4]. CT scan and MRI can be extremely informative and sometimes reveal surprising findings like in this case. Bhullar reports that 26 cases (13\%) were diagnosed as being fabricated or were strongly suspected of being so out of a total of 200 cases of assault examined in a Medical College in Punjab, India over a period of a year. These were brought about to support a false charge of assault [5].

In another study from the same area, 757 medico-legal injury cases were examined to know the extent of fabricated injuries with reference to their nature. Of the above, 159 were opined to be grievous of which $62(38.99 \%)$ were fabricated cut fractures [1]. Khichi., et al. from Pakistan report that out of 130 medico-legal cases in Larkana Division, 50 (38.46\%) were proved to be fabricated. The total number of challenged cases was 130, out of these 50 cases were of fabricated injuries. Of these 50 cases, 46 (92\%) were males and $4(8 \%)$ were female, thus the male to female ratio was approximately 11:1 [6].

The disastrous consequences of investigative agencies and Forensic experts not being able to differentiate between true and 
fabricated wounds need not be emphasized. Usually minor injuries on accessible parts of the body constitute the quantum of fabricated wounds. However, misrepresentation of old healed surgical scars as evidence of a recent assault has scarcely been reported. With this background, we report of an old neuro-surgical craniotomy wound being claimed to be an acute head injury by the patient as is discussed in the present case.

\section{Review of Literature}

Fabricated wounds have been documented in medical literature from throughout the world. Most of the fabricated injuries are caused on accessible part of the body like arms, legs, abdomen, chest, etc [6]. Self-inflicted injuries which are usually nonfatal may be motivated by some form of gain and that the fabrication of injuries to simulate an assault is seen among the self-inflicted injuries $[2]$.

A total of 14 cases with self-inflicted injuries intended to simulate a criminal offence are reported by Karger., et al. Typical characteristics of self-infliction such as superficiality, localization at the anterior aspect, parallel course or avoidance of areas with high sensitivity to pain are substantiated by most of the cases analyzed. However, the majority of cases also comprised atypical features including large hematomas of the extremities, a gaping cut wound, lip laceration, hematoma of the eyelids concussion of the brain, large burn injuries and signs of bonding. Therefore, atypical injuries, which are either found in isolation or in combination with typical injury patterns, cannot exclude self-infliction [7].

Further diagnostic clues for fabricated injuries were put forward by Byard et al who discussed four cases where in the evaluation of injuries due to self-mutilation, one of the most striking findings was the presence of "mirror-image" injuries and/or a "chessboard" pattern of intersecting parallel lines from self-inflicted incised wounds [8].

\section{Case Report}

The case involved a male, aged 30 years who pressed criminal charges and claimed to be a victim of a physical assault that resulted in a head injury. At the accuser's request and mandated by the District Court, a re-evaluation of the case has been conducted in a specialized forensic unit. The medical re-examination took place three months after the initial trauma.

The first examination of the patient was carried out at a peripheral hospital. The medical officer mentioned two 'skin deep' incised wounds over right parietal region of scalp. There was no mention of size, extent and precise location. Radiological examination was advised and a Plain CT Scan of the Head was done at a private facility. The CT scan report showed a supra-tentorial hypo-density in the right parietal area of the brain with oedema and a localized non-heamorrhagic contusion. It also reported a bony defect in the right parietal region with overlying soft tissue swelling. (Figures A,B \& C).

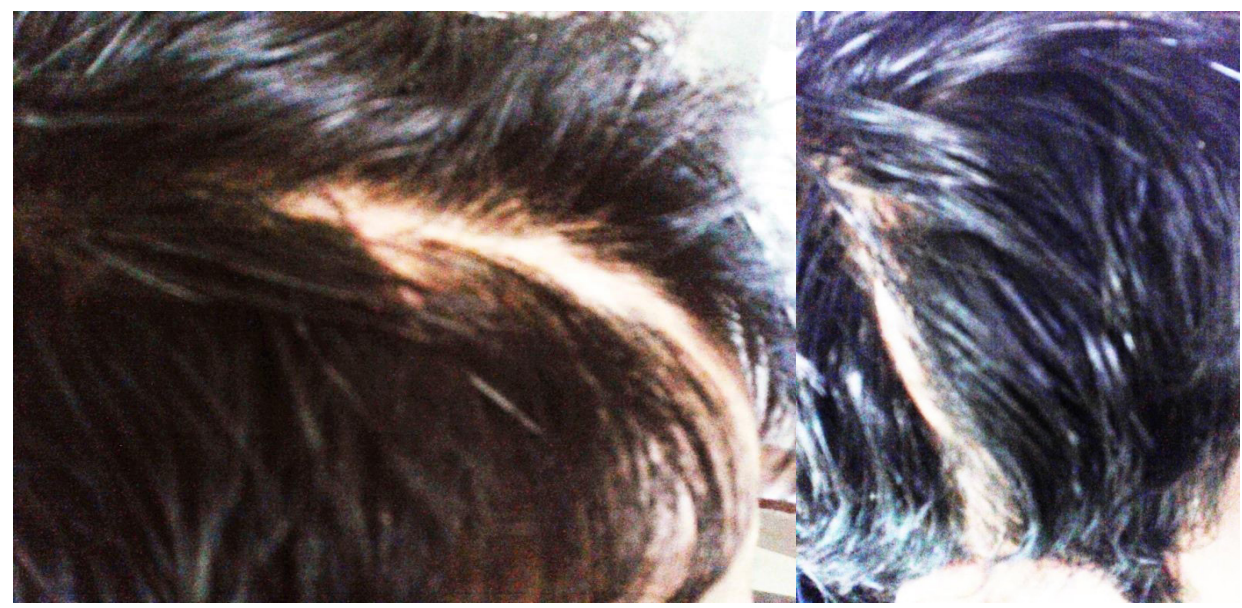

Figures I \& II: The sharp surgical curvilinear scar seen within the scalp hair (Anterior I to Posterior II)

The patient was medically re-examined at our Center three months after the above primary examination wherein a scar of curvilinear incision (surgically made) staring from a point $1 \mathrm{~cm}$ in front of tip of right ear on temporal area of scalp going upwards on parietal area in the same curvilinear manner \& then forwards to a point $7 \mathrm{~cm}$ above mid-point of supra-orbital margin; size 23.5 $\mathrm{x} 0.5 \mathrm{~cm}$ long was noted. This injury did not correspond to any of the injuries mentioned in the primary examination done at the District hospital. (Figures I \& II) Interestingly, the patient refused to get his hair shaved for closer examination of his scalp injury before his radiological investigations.

The radiological examinations (CT scan Head) were repeated at our center and showed multiple burr-hole defects with the craniotomy flap and 3 burr holes, linear hypo-density in the parietal parenchyma; the bony defects of the craniotomy\& gliosis suggestive of a previous surgery. (Figures A1, B1 \& C1). The previous CT scan done at the Private hospital, 3 months earlier were also reviewed and we noted that this CT scan showed similar findings except for soft tissue swelling in the higher cuts which had subsided with passage of time.(Figures A, B \& C). In this case, the patient tried to present an old surgical scar as an injury sustained 
in a recent assault. A curvilinear scar present on the right parieto-temporal area, too clean to be caused by a sharp weapon as claimed was present; and secondly there was a craniotomy flap with 3 burr holes visible in the initial and repeat CT scans. Linear hypo-density in parietal parenchyma suggestive of localized gliosis was seen in both previous and repeat CT films.

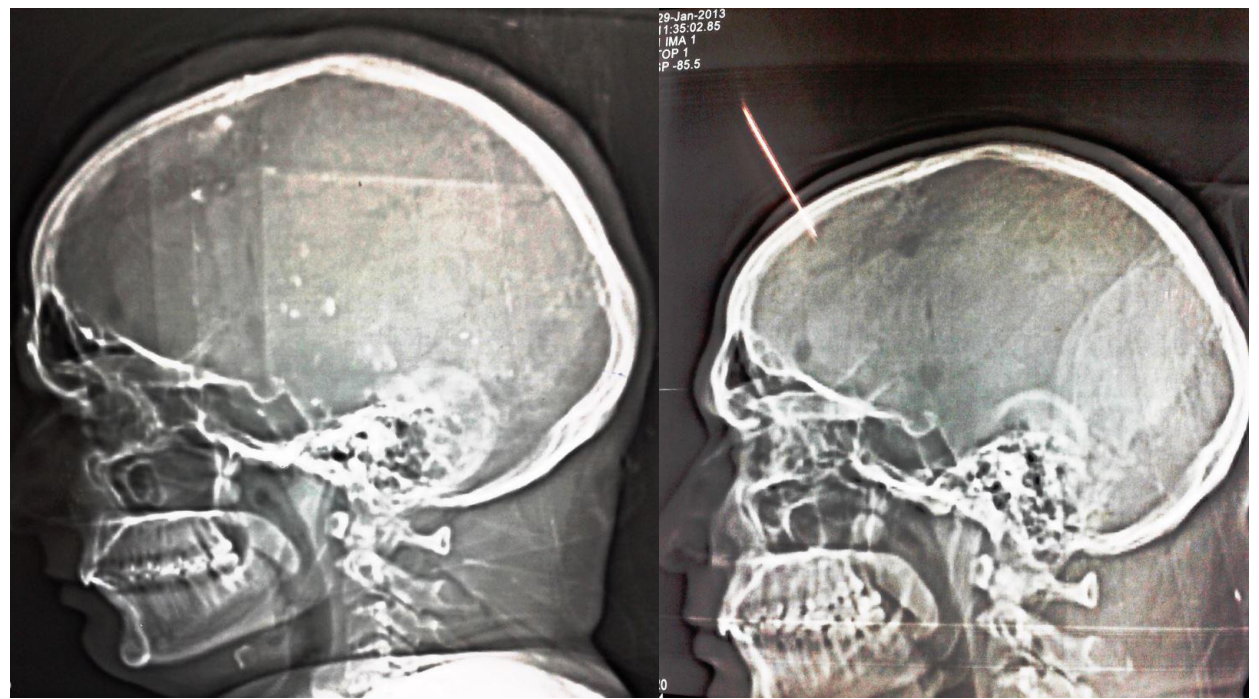

Figures A \& A1: Scanograms of CT showing the craniotomy flap and 3 burr holes in both CT scan films done on $1^{\text {st }}$ October $2012 \& 29^{\text {th }}$ January 2013 (Initial CT Scan \& CT Scan at our center)

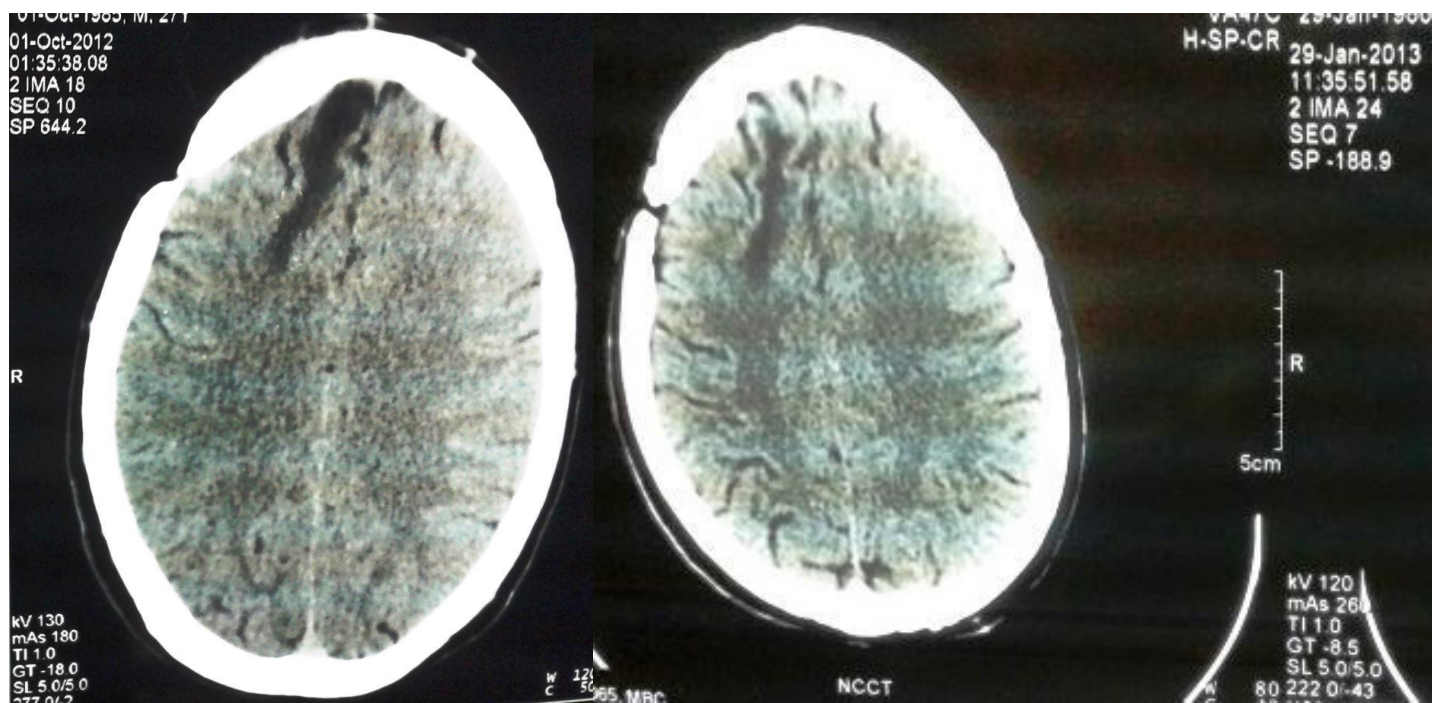

Figures B \& B1: CT scans showing linear Hypodensity in the Parietal parenchyma in both CT scan films done on $1^{\text {st }}$ October $2012 \& 29^{\text {th }}$ January 2013 (Initial CT Scan \& CT Scan at our center)

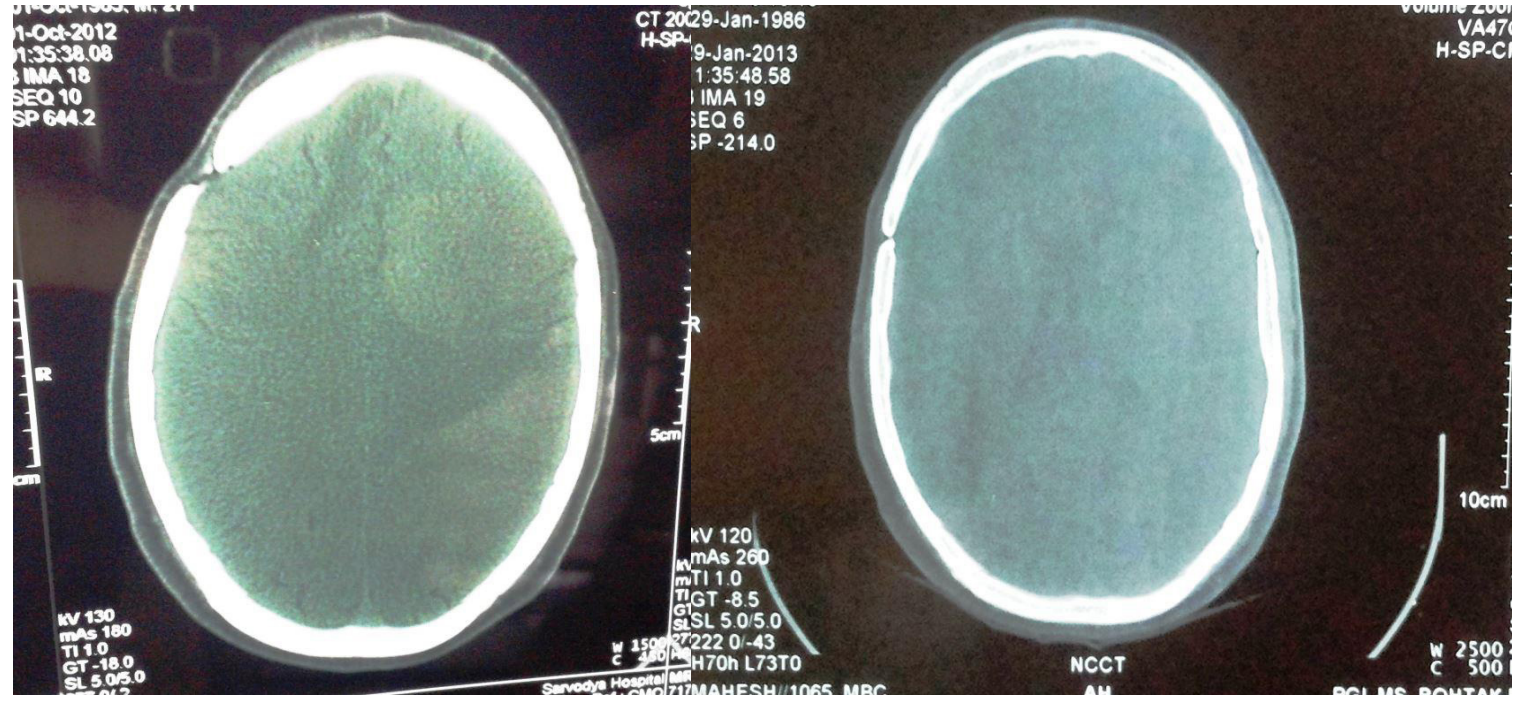

Figures C \& C1: CT scans showing the bony defects of the craniotomy flap in both CT scan films done on $1^{\text {st }}$ October $2012 \& 29^{\text {th }}$ January 2013 (Initial CT Scan \& CT Scan at our center) 


\section{Discussion and Conclusions}

The patient underwent two separate examinations within a space of three months, the first being at a District Hospital by a board of medical officers. The board of doctors there examined the patient clinically and radiologically. They reported two superficial incised wounds on the scalp with a fracture of the underlying right parietal bone of the skull. On the request of the accused persons, a district court ordered for medical re-examination by a board of specialist doctors including the authors. The examination revealed a wrongly diagnosed old craniotomy scar over the scalp with underlying burr holes and craniotomy flap. The discovery of evidence of previous surgery during the second CT scan further emphasizes the paramount importance of comparative radiology in forensic diagnosis.

\section{References}

1. Gorea RK, Gargi J, Aggarwal AD (2007) Incidence and pattern of fabricated injuries. J Punjab Acad For Med Toxicol 7: 11-4.

2. Rajapaksha WRAS, Paranitharan P, Perera WNS (2010) A clinical case of an 'alleged assault': are they fabricated injuries? Sri Lanka Journal of Forensic Medicine, Science \& Law. 1: 32-5.

3. Luber SD, Brady WJ, Brand A, Young J, Guertler AT., et al. (1996) Acute hypoglycemia masquerading as head trauma: A report of four cases. Am J Emer Med 14: 543-7.

4. Gentleman D (2008) Synopsis of Causation: Head Injury. London, UK: Veterans UK \& Ministry of Defence.

5. Bhullar DS (2006) Profile \& Pattern of Fabricated injuries by Mechanical Violence in G.G.S. Medical College, Faridkot (Punjab). J Indian Academy Forensic Medicine 28: 31-4

6. Khichi ZH, Humayun M, Prithiani KK, Akbar QM, Kaheri GQ (2006) Drug Non-Adherence in Type 2 Diabetes Mellitus; Predictors and Associations. J Ayub Med Coll (JAMC) 21: 76-8

7. Karger B, DuChesne A, Ortmann C, Brinkmann B (1997) Unusual self-inflicted injuries simulating a criminal offence. Int J Legal Med 110: $267-72$.

8. Byard Roger W, Gilbert JD, Tsokos M (2007) Symmetrical “Mirror-Image” Injuries and the "Chessboard” Pattern: Useful Markers of Self-Mutilation. Am J For Med Path 28: 255-8. 